\title{
Applying Nonlinear MODM Model to Supply Chain Management with Quantity Discount Policy under Complex Fuzzy Environment
}

\author{
Zhe Zhang ${ }^{1}$, Jiuping $\mathrm{Xu}^{2 *}$ \\ ${ }^{1}$ School Economics \& Management, Nanjing University of Science and Technology (CHINA) \\ ${ }^{2}$ Uncertainty Decision-Making Laboratory, Sicbuan University (CHINA) \\ zhangzhe@,njust.edu.cn,xujiuping@scu.edu.cn
}

Received: January 2014

Accepted: May 2014

\section{Abstract:}

Purpose: The aim of this paper is to deal with the supply chain management (SCM) with quantity discount policy under the complex fuzzy environment, which is characterized as the bifuzzy variables. By taking into account the strategy and the process of decision making, a bifuzzy nonlinear multiple objective decision making (MODM) model is presented to solve the proposed problem.

Design/methodology/approach: The bi-fuzzy variables in the MODM model are transformed into the trapezoidal fuzzy variables by the DMs's degree of optimism $\alpha_{1}$ and $\alpha_{2}$, which are de-fuzzified by the expected value index subsequently. For solving the complex nonlinear model, a multi-objective adaptive particle swarm optimization algorithm (MO-APSO) is designed as the solution method.

Findings: The proposed model and algorithm are applied to a typical example of SCM problem to illustrate the effectiveness. Based on the sensitivity analysis of the results, the bifuzzy nonlinear MODM SCM model is proved to be sensitive to the possibility level $\alpha_{1}$.

Practical implications: The study focuses on the SCM under complex fuzzy environment in SCM, which has a great practical significance. Therefore, the bi-fuzzy MODM model and MOAPSO can be further applied in SCM problem with quantity discount policy. 
Originality/value: The bi-fuzzy variable is employed in the nonlinear MODM model of SCM to characterize the hybrid uncertain environment, and this work is original. In addition, the hybrid crisp approach is proposed to transferred to model to an equivalent crisp one by the DMs's degree of optimism and the expected value index. Since the MODM model consider the bi-fuzzy environment and quantity discount policy, so this paper has a great practical significance.

Keywords: bi-fuzzy variable, nonlinear, multi-objective programming, sensitivity analysis, particle swarm optimization

\section{Introduction}

A supply chain (SC) is a system of facilities and activities that functions to procure, produce, and distribute goods to the customers. Basically, supply chain management (SCM) is a set of approaches utilized to efficiently integrate suppliers, manufacturers, warehouses, and stores, so that merchandise is produced and distributed at the right quantities, to the right locations, and at the right time, in order to minimize system-wide costs (or maximize profits) while satisfying service level requirements (Simchi-Levi, Kaminsky \& Simchi-Levi, 2000). In this situation, SCM has become the foundation for the operations management nowadays (Al-e-hashem, Malekly \& Aryanezhad, 2011). In traditional SCM, the focus of the integration of supply chain network is usually on single objective, i.e., minimum cost or maximum profit. However, in practice, there are no design tasks that are single objective problems. In SC, different members have different conflicting objectives, such as cost and quality, on time delivery and quality, and so on. Chen and Lee (2004) presented a multi-product, multi-stage, and multi-period scheduling model to deal with multiple incommensurable goals for a multi-echelon supply chain network with uncertain market demands and product prices. Altiparmak, Gen, Lin and Paksoy (2006) designed a new solution procedure based on genetic algorithms to find the set of Pareto-optimal solutions for multi-objective supply chain network problem. In addition, to deal with the multiple objectives and enable the decision maker for evaluating a greater number of alternative solutions, two different weight approaches are implemented in the proposed solution procedure. Torabi and Hassini (2008) proposed a multiobjective possibilistic mixed integer linear programming model (MOPMILP) for integrating procurement, production and distribution planning considering various conflicting objectives simultaneously as well as the imprecise nature of some critical parameters such as market demands, cost/time coefficients and capacity levels. Arikan (2013) considered three objective functions, which were minimization of costs, maximization of quality and maximization of ontime delivery, in the suppliers selection problems of SCM. The application of MODM in SCM will become increasingly extensive and in-depth because SCM has made managers and analysts to shift their focuses from only manufacturing plant to the entities process. 
Another key issue which also worth our attention in SCM is the inevitable uncertainty. Actually, the variables in SCM, such as the market demands, availabilities of raw materials, buyer's cost, are usually uncertain. Traditionally, the subjective uncertainty, i.e. the perception and dissension of decision makers (DMs), in SCM is assumed to be fuzzy. The fuzzy set theory, which was initialized by Zadeh in 1965, can be used to handle the uncertain issues such as demands, external raw material supply delivery, inventory cost, and so on. Giannoccaro, Pontrandolfo and Scozzi (2003) applied the fuzzy sets theory to characteristic the uncertainties associated with both market demand and inventory cost. Wei, Liang and Wang (2007) adopted the fuzzy set theory to resolve the ambiguities involved in assessing SCM alternatives and aggregating the linguistic evaluations. Wang and Shu (2008) developed a fuzzy decision methodology that provided an alternative framework to handle supply chain uncertainties and to determine supply chain inventory strategies, while there was lack of certainty in data or even lack of available historical data. Tabrizi and Razmi (2013) proposed a mixed-integer non-linear mathematical model in which the uncertainties were represented by the fuzzy set theory, and applied an interactive resolution method to provide the decision maker with alternative decision plans in regard to the different satisfaction degrees. In practice, however, we may face a complex fuzzy environment in the practical SCM. For example, in order to collect the data of inventory cost, some investigations and surveys are made to the different experienced managers (i.e., $m=1,2, \ldots, M$, where $m$ is the index of managers). Instead of the exact parameters, the managers can describe the parameters as an interval $\left[I_{m}, r_{m}\right]$ with the most possible value $p_{m}$ (i.e., a fuzzy variable $\left(I_{m}, p_{m}, r_{m}\right)$ ), such as "the maximal inventory cost is between 6.35 and 8.13 hundred dollars per week, and the most possible cost is 7.62 hundred dollars". Since different managers have different opinions for the parameters, so the minimum value of all $I_{m}$ (denoted as $a$ ) and the maximal value of all $r_{m}$ (denoted as $b$ ) are selected as the left border and the right border, respectively. Meanwhile, by comparing the most possible values, we can also select the minimal value as its left border $\left(a_{p}\right)$ and the maximal value as the right border $\left(b_{p}\right)$ for $p_{m}$. In addition, after considering the professional advice of all managers, the director declared the most likely value of $p_{m}$ is $m_{p}$. It means that $p_{m}$ is also a fuzzy variable which can be characterized as $p_{m}=\left(a_{p}, m_{p}, b_{p}\right)$. Hence, the inventory cost is a fuzzy variable taking a fuzzy parameter, i.e., a bi-fuzzy variable $\left(a, p_{m}, b\right)$, where $p_{m}=\left(a_{p}, m_{p}, b_{p}\right)$. Hence, the bi-fuzzy variable, which was initialized by Liu in 2002, can be a useful tool which is able to deal with this complex uncertainty. In fact, the bi-fuzzy variable has been successfully applied in many areas, such as vendor selection problem (Xu \& Yan, 2011), inventory problems (Liu \& Xu, 2006), portfolio selection problems (Yan, 2009), and so on. The research mentioned above proved that the bi-fuzzy variable could effectively cope with the complex fuzzy environment. So far, no attempt has been made on considering decision making process in the bi-fuzzy environment for SCM. Hence, there is a strong motivation for this study. 
The remainder of this paper is organized as follows. In section 2, some preliminaries about the bi-fuzzy theory are presented. Then a bi-fuzzy nonlinear MODM SCM model with quantity discount policy is proposed in section 3. The details of an approach used in transforming bifuzzy variables into the fuzzy variables are also presented, and then the expected value operator is employed to deal with the fuzzy variables. In section 4, a multi-objective adaptive particle swarm optimization algorithm (MOBL-APSO) is utilized to resolve the nonlinear MODM model. The effectiveness of the proposed model and algorithm is proven by the practical application in section 5. Finally, in section 6, concluding remarks and further research are outlined.

\section{Preliminaries}

Some basic knowledge of fuzzy set theory, bi-fuzzy variable and bi-fuzzy MODM model will be introduced in this section.

Definition 2.1. (Zadeh, 1965) Given a domain $X$. If $\tilde{A}$ is a fuzzy subset of $X$, for any $x \in X$

$$
\mu_{\tilde{A}}: X \rightarrow[0,1], x \rightarrow \mu_{\tilde{A}}(x)
$$

$\mu_{\tilde{A}}$ is named a membership function of $x$ with respect to $\tilde{A} . \mu_{\tilde{A}}(x)$ denotes the grade to each point in $X$ with a real number in the interval $[0,1]$ that represents the grade of membership of $x$ in $A . \tilde{A}$ is a fuzzy set and described as $\tilde{A}=\left\{\left(\mathrm{x}, \mu_{\tilde{A}}(x)\right) \mid x \in X\right\}$. If $F$ is a fuzzy number (set) with degree of membership $\mu_{F}(u)$ of an element $u$ in $F$, then $\mu_{F}(u)$ represents the degree of possibility that a parameter $x$ has a value $u$. Thus, the nearer the value of $\mu_{\tilde{A}}(x)$ is unity, the higher the grade of membership of $x$ in $\tilde{A}$.

Definition 2.2. (Zadeh, 1978) Let $\tilde{A}$ be a fuzzy set which defined on $X$. If $\alpha$ is possibility level and $0 \leq \alpha \leq 1, \tilde{A}_{\alpha}$ consist of all elements whose degrees of membership in $\tilde{A}$ are greater than or equal to $\alpha$,

$$
\tilde{A}_{\alpha}=\left\{x \in X \mid \mu_{\tilde{A}}(x) \geq a\right\}
$$

then $\tilde{A}_{\alpha}$ is called the $\alpha$-level set of fuzzy set $\tilde{A}$.

Definition 2.3. (Dubois \& Prade, 1988) Let $\Theta$ be a nonempty set, $P(\Theta)$ be the power set of $\Theta$, and Pos be a possibility measure. Then the triplet $(\Theta, P(\Theta)$, Pos) is a possibility space. 
Definition 2.4. (Nahmias, 1978) A fuzzy variable is a function from a possibility space $(\Theta$, $P(\Theta)$, Pos) to the real line $R$.

Definition 2.5. (Liu, 2002) A bi-fuzzy variable is a function from a possibility space $(\Theta, P(\Theta)$, Pos) to a collection of fuzzy variables.

Roughly speaking, a bi-fuzzy variable is a fuzzy variable defined on the universal set of fuzzy variables. For example, let $\tilde{\xi}=(1, \tilde{\rho}, 4)$, where $\tilde{\rho}$ is a fuzzy variable with membership function $\mu_{\bar{\rho}}=(2-|x-1|) \vee 0$. Then $\tilde{\xi}$ is a bi-fuzzy variable obviously (Liu, 2002).

Based on the definitions above, the bi-fuzzy MODM model can be stated as:

$$
\left\{\begin{array}{l}
\max \left\{\tilde{\tilde{c}}_{1} x, \tilde{\tilde{c}}_{2} x, \cdots, \tilde{\tilde{c}}_{K} x\right\} \\
\text { s.t. } x \in X=\left\{x \in R^{n} \mid \tilde{\tilde{A}} x \leq b, x \geq 0\right\}
\end{array}\right.
$$

Where $\tilde{\tilde{c}}_{k}=\left(\tilde{\tilde{c}}_{k 1}, \tilde{\tilde{c}}_{k ?}, \cdots, \tilde{\tilde{c}}_{k n}\right)(k=1,2, \cdots, K), \tilde{\tilde{A}}=\left(\tilde{\tilde{A}}_{1}, \tilde{\tilde{A}}_{2}, \cdots, \tilde{\tilde{A}}_{m}\right)^{T}, \tilde{A}_{i}=\left(\tilde{a}_{i 1}, \tilde{a}_{i 2}, \cdots, \tilde{a}_{i n}\right)$ are bi-fuzzy vectors, and $b=\left(b_{1}, b_{2}, \cdots, b_{m}\right)^{T}$.

Definition 2.6. (Luhandjula, 1987) Let $\alpha_{1}=\left(\alpha_{1_{1}}, \alpha_{1_{2}}, \cdots, \alpha_{1_{m}}\right)^{T}$ be the possibility level vector, $\alpha_{1_{i}} \in[0,1], x \in R^{n}$, and if

$$
\operatorname{poss}\left(\tilde{\tilde{A}}_{i} x \leq b_{i}, \tilde{\tilde{c}}_{k} x\right) \geq \alpha_{1_{i}}, i=1,2, \cdots, m, k=1,2, \cdots, K
$$

then $x$ is $\alpha_{1}$-possible feasible solution to (1). All $\alpha_{1}$-possible feasible solutions are $\alpha_{1}$-possible feasible set $X_{\alpha_{1}}$ of the model (1).

Consider the form of model (1) as:

$$
\max _{x \in D}\left\{\tilde{\tilde{c}}_{1} x, \tilde{\tilde{c}}_{2} x, \cdots, \tilde{\tilde{c}}_{K} x, \tilde{\tilde{A}}_{i} x\right\}, i=1,2, \cdots, m
$$

Then, let $\alpha_{2}$ be a possibility level, $\alpha_{2} \in[0,1], D \in R^{n}$, and $x^{\prime} \in D$. If do not exist $x \in D$ and $k \in 1,2, \cdots, k, x$ satisfy

$\operatorname{poss}\left(\tilde{\tilde{c}}_{1} x \geq \tilde{\tilde{c}}_{1} x^{\prime}, \cdots, \tilde{\tilde{c}}_{k-1} x \geq \tilde{\tilde{c}}_{k-1} x^{\prime}, \tilde{\tilde{c}}_{k} x \geq \tilde{\tilde{c}}_{k} x^{\prime}, \tilde{\tilde{c}}_{k+1} x \geq \tilde{\tilde{c}}_{k+1} x^{\prime}, \cdots, \tilde{\tilde{c}}_{K} x \geq \tilde{\tilde{c}}_{K} x^{\prime}, \tilde{\tilde{A}}_{K+i} x \geq \tilde{\tilde{A}}_{K+i} x^{\prime}\right) \geq \alpha_{2} x^{\prime}$ is $\alpha_{2}$-possible efficient solution of the model (2) (Luhandjula, 1987). 
Definition 2.7. (Luhandjula, 1987) Let $x^{\prime} \in X$, if $x^{\prime}$ is the problem

$$
\left\{\begin{array}{l}
\max \left\{\tilde{\tilde{c}}_{1} x, \tilde{\tilde{c}}_{2} x, \cdots, \tilde{\tilde{c}}_{K} x, \tilde{\tilde{A}}_{i} x\right\} \\
\text { s.t. } x \in X_{\alpha_{1}}
\end{array}\right.
$$

$\alpha_{1}$-possible efficient solution, and then $x^{\prime}$ is $\left(\alpha_{1}, \alpha_{2}\right)$-satisfied solution of the model (1).

Actually, in order to solve model (1) and find the $\left(\alpha_{1}, \alpha_{2}\right)$-satisfied solution, the following MODM model should be considered:

$$
\left\{\begin{array}{l}
\max \left\{\left(\tilde{\tilde{c}}_{1}\right)_{\alpha_{1}} x,\left(\tilde{\tilde{c}}_{2}\right)_{\alpha_{1}} x, \cdots,\left(\tilde{\tilde{c}}_{K}\right)_{\alpha_{1}} x,\left(\tilde{\tilde{A}}_{i}\right)_{\alpha_{1}} x\right\} \\
\text { s.t. } x \in X_{\alpha_{1}}
\end{array}\right.
$$

Where $\left(\tilde{\tilde{c}}_{k}\right)_{\alpha_{1}},\left(\tilde{\tilde{A}}_{i}\right)_{\alpha_{1}}$ is $\alpha_{1}$-level set of bifuzzy variables $\tilde{\tilde{c}}_{k}, \tilde{\tilde{A}}_{i}$.

Theorem 1. (Xu \& Liu, 2008) $x^{\prime}$ is the $\left(\alpha_{1}, \alpha_{2}\right)$-satisfied solution of the model (1) if and only if $x^{\prime}$ is the efficient solution of the model (4).

\section{Bi-fuzzy non-linear MODM model for SCM}

Traditionally, there are three stages in the supply chain, including procurement, production and distribution. The general structure of a typical supply chain is outlined in Figure 1 . For simplifying the presentation, only the manufacturers, distribution centers, and retailers are considered in this paper.

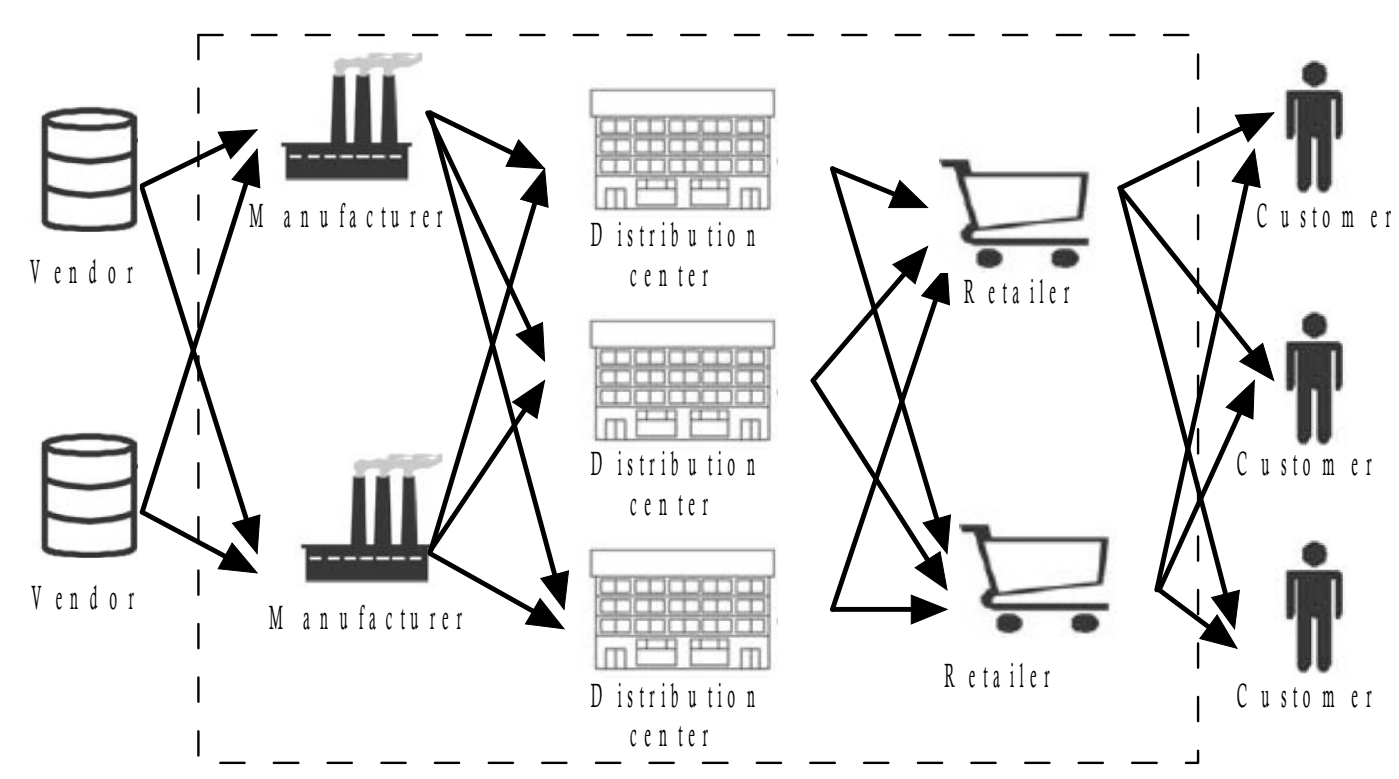

Figure 1 . The schema of supply chain 


\subsection{Modelling}

Since treating the nonlinear functions is difficult, some SCM models assume that the prices of materials, production, inventory, and transportation are constant. However, this assumption is far beyond the practical situation. The vendors usually offer quantity discounts to encourage the buyers to order more, and the producer intends to discount the unit production cost if the amount of production is large. In this case, the quantity discount policy should be considered.

Note that the cost (price) variables can be expressed as a function of quantity $Q$, and the multiple breakpoint discount function is a general form usable in practice (see Figure 2 ). The function can be represented as:

$$
\tilde{\tilde{C}}(Q)=\left\{\begin{array}{c}
\tilde{\tilde{C}}_{1}+s_{1}\left(Q-Q_{1}\right), \text { if } Q_{1} \leq Q<Q_{2} \\
\tilde{\tilde{C}}_{2}+s_{2}\left(Q-Q_{2}\right), \text { if } Q_{2} \leq Q<Q_{3} \\
\vdots \\
\tilde{\tilde{C}}_{n-1}+s_{n-1}\left(Q-Q_{n-1}\right), \text { if } Q_{n-1} \leq Q<Q_{n}
\end{array}\right.
$$

where $s_{i}$ is the slope when the quantity ordered is between $Q_{i}$ and $Q_{i+1}$, and $n$ means that there are $n-1$ line segments in $\tilde{\tilde{C}}(Q)$.

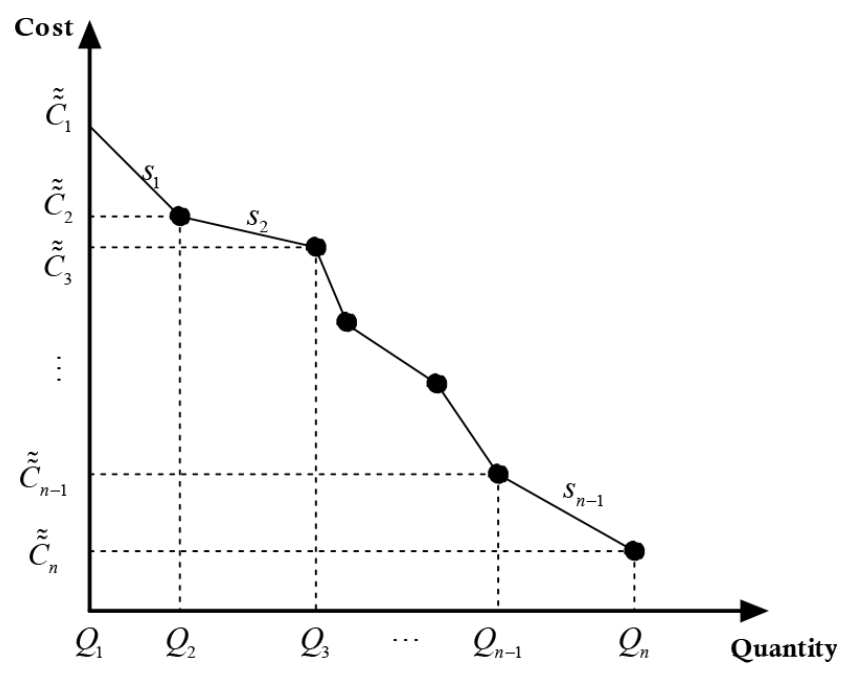

Figure 2. Multiple breakpoint discount function

In this subsection, a non-linear MODM model for SCM under complex fuzzy environment is developed. The following notation is used. 


\subsubsection{Notion}

$Q 1_{m p}^{t} \quad:$ Ordered quantity of the $p$ th product from the $m$ th manufacturer in the $t$ th period

$Q 2_{m p d}^{t} \quad$ : Quantity of the $p$ th product transported from the $m$ th manufacturer to the $d$ th distribution center in the th period

$Q 3_{p d}^{t} \quad:$ Quantity of the $p$ th product in the $d$ th distribution center in the th period

$Q 4_{p d r}^{t} \quad:$ Quantity of the $p$ th product transported from the $d$ th distribution center to the $r$ th retailer in the th period

$\tilde{\tilde{P}}_{\cos \mathrm{t}_{m \bar{x}}^{t}}$ : Unit procurement cost of the $p$ th product from the $m$ th manufacturer in the $t$ th period

$\tilde{\tilde{T}} \cos 11_{m p d}^{t}:$ Unit transportation cost of the $p$ th product from the $m$ th manufacturer to the $d$ th distribution center in the $t$ th period

$\tilde{\tilde{T}} \cos 2_{p d r}^{t}$ : Unit transportation cost of the $p$ th product from the $d$ th distribution center to the $r$ th retailer in the $t$ th period

$\tilde{\tilde{C}} \cos _{p d}^{t} \quad$ : Unit stock carrying cost of the pth product in the $d$ th distribution center in the th period

$D_{*_{p}}^{t} \quad$ : Short safe inventory level in the $m$ th manufacturer, $d$ th distribution center, $r$ th retailer in the $t$ th period

$S I_{*_{p}} \quad$ : Safe inventory quantity in the $m$ th manufacturer, $d$ th distribution center, $r$ th retailer in the $t$ th period

$I_{*_{p}}^{t} \quad$ : Inventory level of the $p$ th product in the $m$ th manufacturer, $d$ th distribution center, $r$ th retailer in the th period

MIC* : Maximum inventory capacity of the $m$ th manufacturer, $d$ th distribution center, $r$ th retailer

$L T_{m \dot{d}} \quad$ : Lead time from the $m$ th manufacturer to the $d$ th distribution center and $L T_{m d} \leq T$

$L T_{d r} \quad$ : Lead time from the $d$ th distribution center to the $r$ th retailer and $L T_{d r} \leq T$

$S_{m p}^{t}(\max ):$ Maximum supplied level of the $p$ th product from the $m$ th manufacturer in the $t$ th period

$I_{p d}^{t}(\max )$ : Maximum inventory level of the $p$ th product in the $d$ th distribution center in the th period 


\subsubsection{Model Formulation}

Based on the requirement of the DM's objectives, we will develop a bi-fuzzy non-linear MODM model for SCM as follows.

\subsubsection{Objective functions}

The first objective is to minimize the total cost, which includes procurement cost, transportation cost, and inventory cost. Basically, there are four kinds of costs involved in this model: product procurement cost from manufacturers, transportation cost from manufacturers to distribution centers, inventory cost in distribution centers, and transportation cost from distribution centers to retailers. So the total cost can be described as:

$$
\min C=\sum_{t}\left(\sum_{m, p} \tilde{\tilde{P}} \cos \mathrm{t}_{m p}^{t} \times Q 1_{m p}^{t}+\tilde{\tilde{T}} \cos \mathrm{t}_{m p d}^{t} \times Q 2_{m p d}^{t}+\tilde{\tilde{C}} \cos \mathrm{t}_{p d}^{t} \times Q 3_{p d}^{t}+\tilde{\tilde{T}} \cos \mathrm{t}_{p d r}^{t} \times Q 4_{p d r}^{t}\right)
$$

Where $\tilde{\tilde{P}} \cos t_{m p}^{t}, \tilde{\tilde{T}} \cos \mathrm{tl}_{m p d r}^{t}, \tilde{\tilde{T}} \cos \mathrm{t}_{p d r}^{t}, \tilde{\tilde{C}} \cos \mathrm{t}_{p d}^{t}$ are bi-fuzzy variables.

Furthermore, the second objective is to maximize the average safe inventory levels. The safe inventory level of the $m$ th manufacturer in the $t$ th period is defined as the expected average percentage of 1 less the ratio of short safe inventory level of product $p$ of manufacturer $m$ at period $t\left(D_{m p}^{t}\right)$, over the safe inventory quantity of product $p$ of manufacturer $m\left(S I_{m p}\right)$. Similar definitions are also applied to distribution centers and retailers. So we develop all the participants' safe inventory levels as:

$$
\max S=\sum_{p, t}\left(1-\frac{D_{m p}^{t}}{S I_{m p}}\right)+\sum_{p, t}\left(1-\frac{D_{d p}^{t}}{S I_{d p}}\right)+\sum_{p, t}\left(1-\frac{D_{r p}^{t}}{S I_{r p}}\right)
$$

\subsubsection{Constraints}

Since the amount of the $p$ th product transported from the $m$ th manufacturer to all distribution centers must be equal to the total amount ordered from the $m$ th manufacturer in the th period for each manufacturer, so

$$
Q 1_{m p}^{t}=Q 2_{m p d}^{t}
$$

The stock quantity of the $p$ th product at the beginning of the th period, plus the total amount flowing in from all manufacturers and the stock quantity of the $p$ th product in the $(t+1)$ th period, and this value should be equal to the total amount flowing out to all distribution centers, here, 


$$
Q 2_{m}^{t-L T_{m d}}+Q 3_{p d}^{t}+Q 3_{p d}^{t+1}=Q 4_{p d r}^{t-L T_{d r}}
$$

The total amount of the pth product shipped from distribution centers to retailers must be equal to the total final demands in the th period, hence:

$$
\sum_{d} Q 4_{p d r}^{t-L T_{d r}}=D_{p r}^{t}
$$

The maximum supplied quantity of manufacturers and inventory level of distribution centers are given:

$$
Q \mathrm{1}_{m p}^{t} \leq S_{m p}^{t}(\max ), Q 3_{p d}^{t} \leq I_{p d}^{t}(\max )
$$

By making the short safe inventory level of a product to be zero if inventory level is greater than safe inventory quantity, or to be the difference of safe inventory quantity and inventory level if inventory level is smaller than safe inventory quantity, here:

$$
S I_{*_{p}}-I_{*_{p}}^{t} \leq D_{*_{p}}^{t}
$$

Based on the discussion above, by integrating the Equations (6) (12), a bi-fuzzy nonlinear MODM model for SCM is developed as:

$$
\begin{aligned}
& \min C=\sum_{t}\left(\sum_{m, p} \tilde{\tilde{P}} \cos \mathrm{t}_{m p}^{t} \times Q 1_{m p}^{t}+\tilde{\tilde{T}} \cos \mathrm{t}_{m p d}^{t} \times Q 2_{m p d}^{t}+\tilde{\tilde{C}} \cos t_{p d}^{t} \times Q 3_{p d}^{t}+\tilde{\tilde{T}} \cos 2_{p d r}^{t} \times Q 4_{p d r}^{t}\right) \\
& \max S=\sum_{p, t}\left(1-\frac{D_{m p}^{t}}{S I_{m p}}\right)+\sum_{p, t}\left(1-\frac{D_{d p}^{t}}{S I_{d p}}\right)+\sum_{p, t}\left(1-\frac{D_{r p}^{t}}{S I_{r p}}\right) \\
& \text { s.t. }\left\{\begin{array}{l}
Q 1_{m p}^{t}=Q 2_{m p d}^{t} \\
Q 2_{m}^{t-L T_{m d}}+Q 3_{p d}^{t}+Q 3_{p d}^{t+1}=Q 4_{p d r}^{t-L T_{d r}} \\
\sum_{d} Q 4_{p d r}^{t-L T_{d r}}=D_{p r}^{t} \\
Q 1_{m p}^{t} \leq S_{m p}^{t}(\max ) \\
Q 3_{p d}^{t} \leq I_{p d}^{t}(\max ) \\
S I_{* p}-I_{* p}^{t} \leq D_{*_{p}}^{t} \\
*=\{m, d, r\}
\end{array}\right.
\end{aligned}
$$

\subsection{Dealing with the bi-fuzzy variable}

Since some bi-fuzzy variables are involved in the proposed MODM model (13), and the costs and the quantity discount functions are variable, so it is very hard to be solved. In this case, considering the optimistic-pessimistic attitude of DMs, a hybrid crisp approach is employed to transfer the bi-fuzzy model to an equivalent one. This method transforms the bi-fuzzy variable into a $\left(\alpha_{1}, \alpha_{2}\right)$-level trapezoidal fuzzy variable at first, and then de-fuzzified the trapezoidal fuzzy variable by an expected value operator. 
Denoted the bi-fuzzy variable as $\tilde{\xi}=\left(a_{I}, \tilde{\rho}, a_{R}\right)$, where is $\tilde{\rho}=\left(\rho_{L}, \rho_{0}, \rho_{R}\right)$ a triangular fuzzy variable with membership function $\mu_{\tilde{\rho}}(x)$ (See Figure 3 ).

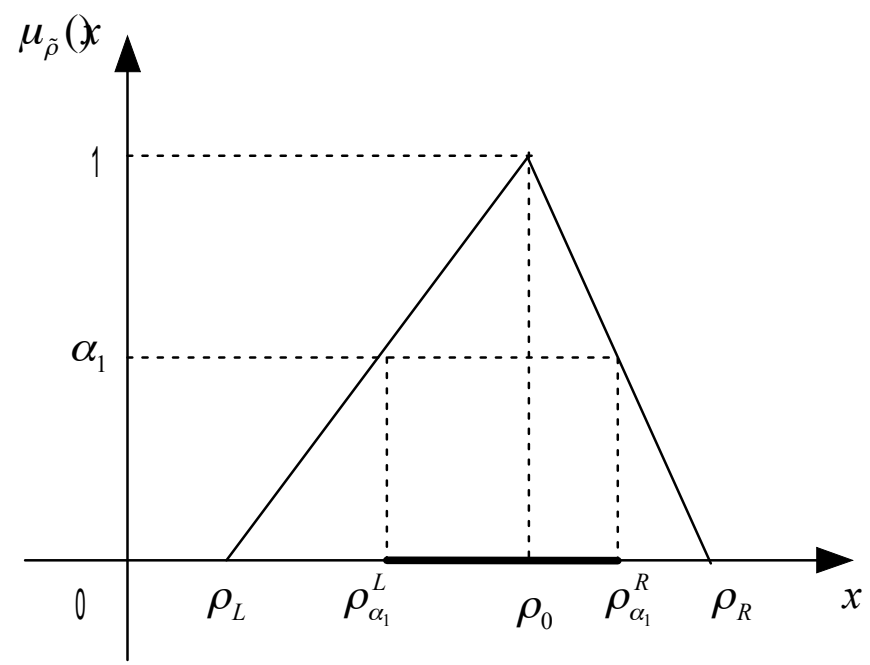

Figure 3. The membership function of $\tilde{\rho}$

Following the definition 2.2, $\alpha_{1}$-level sets of $\tilde{\rho}$ are:

$$
\tilde{\rho}_{\alpha_{1}}=\left[\rho_{\alpha_{1}}^{L}, \rho_{\alpha_{1}}^{R}\right]=\left\{x \in U \mid \mu_{\tilde{\rho}}(x) \geq \alpha_{1}\right\}
$$

where $\rho_{\alpha_{1}}^{L}=\rho_{L}+\alpha_{1}\left(\rho_{0}-\rho_{L}\right)$ and $\rho_{\alpha_{1}}^{R}=\rho_{R}-\alpha_{1}\left(\rho_{R}-\rho_{0}\right)$. The parameter $\alpha_{1} \in[0,1]$ here reflects decision-maker's degree of optimism. In addition, $a_{L}, a_{R}, \rho_{L}, \rho_{R}, \rho_{0}$ can be estimated by collected data and professional experience using statistical methods.

Thus, the bi-fuzzy variable $\tilde{\xi}=\left(a_{L}, \tilde{\rho}, a_{R}\right)$ is transferred as a class of triangular fuzzy numbers, see Figure 4. Subsequently, for the given possibility level $\alpha_{2}$, we can get the $\alpha_{2}$-level set of these triangular fuzzy numbers as Figure 5.

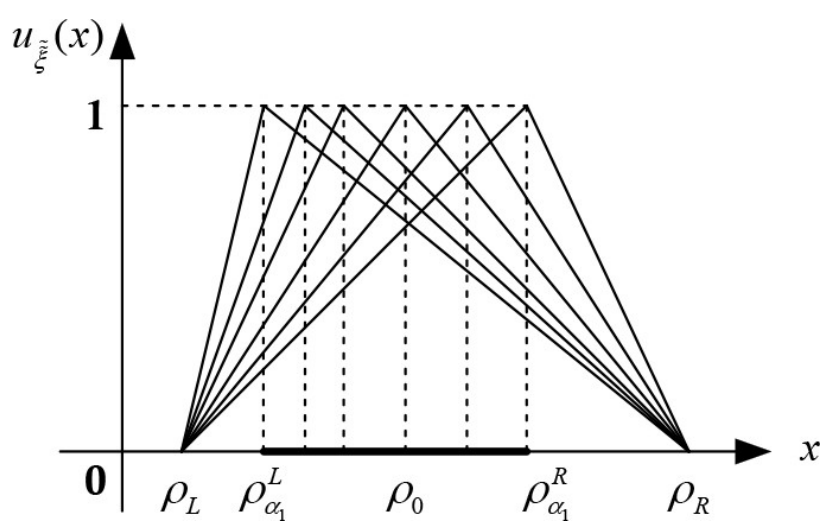

Figure 4. Step 1. Transfer the bi-fuzzy variable to a class of triangular fuzzy numbers

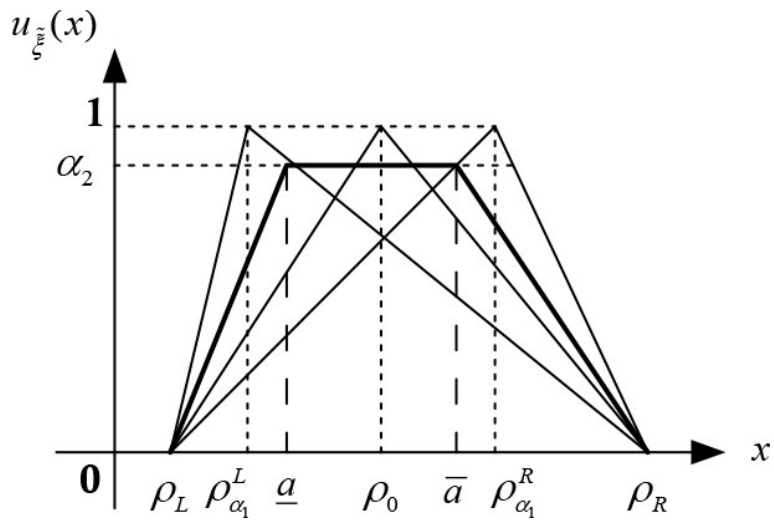

Figure 5. Step 2. Transfer the bi-fuzzy variable to a trapezoidal fuzzy number 
From Figure 5, we can see that the bi-fuzzy variable is transferred as a trapezoidal fuzzy variables $\tilde{\xi} \rightarrow \tilde{\xi}_{\left(\alpha_{1}, \alpha_{2}\right)}=\left(a_{L}, \underline{a}, \bar{a}, a_{R}\right)$, where

$$
\underline{a}=a_{R}-\alpha_{2}\left(a_{R}-\rho_{\alpha_{1}}^{L}\right) \text { and } \bar{a}=a_{L}+\alpha_{2}\left(\rho_{\alpha_{1}}^{R}-a_{L}\right)
$$

Finally, a new measure with an optimistic-pessimistic adjustment index Me, which is proposed by Xu and Zhou (2011) for dealing with the trapezoidal fuzzy variable, is employed. The measure Me can evaluate a confidence degree that a fuzzy variable takes values in an interval, and the expected value of the trapezoidal fuzzy variable can be obtained by Me as (Xu \& Zhou, 2011):

$$
E^{M e}\left[\tilde{\xi}_{\left(\alpha_{1}, \alpha_{2}\right)}=\left(a_{L}, \underline{a}, \bar{a}, a_{R}\right)\right]=\frac{1-\lambda}{2}\left(a_{L}+\underline{a}\right)+\frac{\lambda}{2}\left(\bar{a}+a_{R}\right)
$$

where $\lambda$ is the optimistic-pessimistic index of DMs, and $\lambda=1$ indicates that the best case has the maximal chance to happen, while $\lambda=0$ is opposite.

Based on the above hybrid method, the bi-fuzzy MODM model (13) can be transformed into an equivalent crisp one.

\section{Multiple Objective Adaptive PSO (MO-APSO)}

Since model (13) are nonlinear, so a multi-objective adaptive particle swarm optimization algorithm (MO-APSO) is designed as the solution method. Particle swarm optimization (PSO) algorithm, which was first proposed by Kennedy and Eberhart in 1995, is an effective tool in solving optimization problems because of the superior search performance and fast convergence. PSO simulates the social behaviors such as birds flocking to a promising position for certain objectives in a multi-dimensional space (Kennedy \& Eberhart, 2001). In PSO, an $n$-dimensional position of a particle represents a solution, and the particles fly through the problem space following the current optimum particles. The updating mechanism of particle is:

$$
\begin{aligned}
& v_{l d}(\tau+1)=w(\tau) v_{l d}(\tau)+c_{p} r_{1}\left[p_{l d}^{\text {best }}(\tau)-p_{l d}(\tau)\right]+c_{g} r_{2}\left[p_{g d}^{\text {best }}(\tau)-p_{l d}(\tau)\right] \\
& p_{l d}(\tau+1)=p_{l d}(\tau)+v_{l d}(\tau+1)
\end{aligned}
$$

where $v_{l d}(\tau+1)$ is the velocity of I particle at the $d$ dimension in the $\tau$ iteration, $w$ is an inertia weight, $p_{I d}(\tau)$ is the position of $/$ particle at the $d$ dimension, $r_{1}$ and $r_{2}$ are random numbers in the range $[0,1], c_{p}$ and $c_{g}$ are personal and global best position acceleration constant respectively, meanwhile, $p_{l d}^{\text {best }}$ and $p_{g d}^{\text {best }}$ are personal and global best position of I particle at the $d$ dimension.

As PSO can be implemented easily and effectively, the researchers also consider PSO as a very strong competitor to other algorithms in solving multi-objective decision making problems 
(Coello \& Lechuga, 2002). Some papers reported in the literature have extend PSO to multi-objective problems, such as Garg and Sharma (2013), Tavakkoli-Moghaddam, Azarkish and Sadeghnejad-Barkousaraie (2011), Zhang, Shao, Li and Gao (2009), and so on. The improved PSO utilizes Pareto dominance to determine the flight direction and maintains previously found non-dominated vectors in a global repository (Coello \& Lechuga, 2002). All the particles are compared with each other and the non-dominated particles are stored in the repository. The position of particle is updated by:

$$
v_{l d}(\tau+1)=w(\tau) v_{l d}(\tau)+c_{p} r_{1}\left[p_{l d}^{\text {best }}(\tau)-p_{l d}(\tau)\right]+c_{g} r_{2}\left[R E P_{h}(\tau)-p_{l d}(\tau)\right]
$$

here, $\operatorname{REP}_{h}(\tau)$ is the positions of the particles that represent non-dominated vectors in the repository, i.e., several equally good non-dominated solutions stored in the external repository instead of global best position.

The solution approach proposed in this paper combines multi-objective PSO with Pareto archived evolution strategy (PAES), which is one of Pareto-based approaches to update the best position (Knowles \& Corne, 2000). This approach employs a truncated archive, which is used to separate the objective space into a number of hypercubes, to store the elite individuals. Based on the density, every hypercube has its own score. After selecting the best for particles based on roulette wheel selection, the particle is selected uniformly. More details of PAES are as:

\section{PAES Procedure}

generate initial random solution $P_{/}(\tau)$ and add it to the archive

update $P_{l}(\tau)$ to generate $P_{l}(\tau+1)$

if $P_{l}(\tau)$ dominates $P_{l}(\tau+1)$

discard $P_{/}(\tau+1)$

else if $P_{/}(\tau+1)$ dominates $P_{/}(\tau)$

replace $P_{/}(\tau)$ with $P_{/}(\tau+1)$ and add $P_{/}(\tau+1)$ to the archive

else if $P_{/}(\tau+1)$ is dominated by any member in the archive

discard $P_{l}(\tau+1)$

else if $P_{/}(\tau+1)$ dominates any member in the archive replace it with $P_{/}(\tau+1)$

else if

the archive is not full

add $P_{/}(\tau+1)$ to the archive

if $P_{/}(\tau+1)$ is in a less crowded region than $P_{/}(\tau)$ in the archive accept $P_{/}(\tau+1)$ as the new current solution

else

maintain $P_{l}(\tau)$ as the current solution

else

if $P_{l}(\tau+1)$ is in a less crowded region than any other member 
add $P_{l}(\tau+1)$ to the archive, and remove a member of the archive from the

most crowded region

if $P_{l}(\tau+1)$ is in a less crowed region than $P_{l}^{\text {best }}(\tau)$

accept $P_{l}(\tau)$ as the new current solution

\section{else}

maintain $P_{l}(\tau)$ as the current solution

else

do not add $P_{/}(\tau+1)$ to the archive

until the termination criterion is reached, otherwise return to line 2

In addition, based on PAES, in the updating mechanism, $w(\tau)=w(T)+\frac{\tau-T}{1-T}[w(1)-w(T)]$ (Shi \& Eberhart, 1998). The overall procedure of MO-APSO is presented in Figure 6.

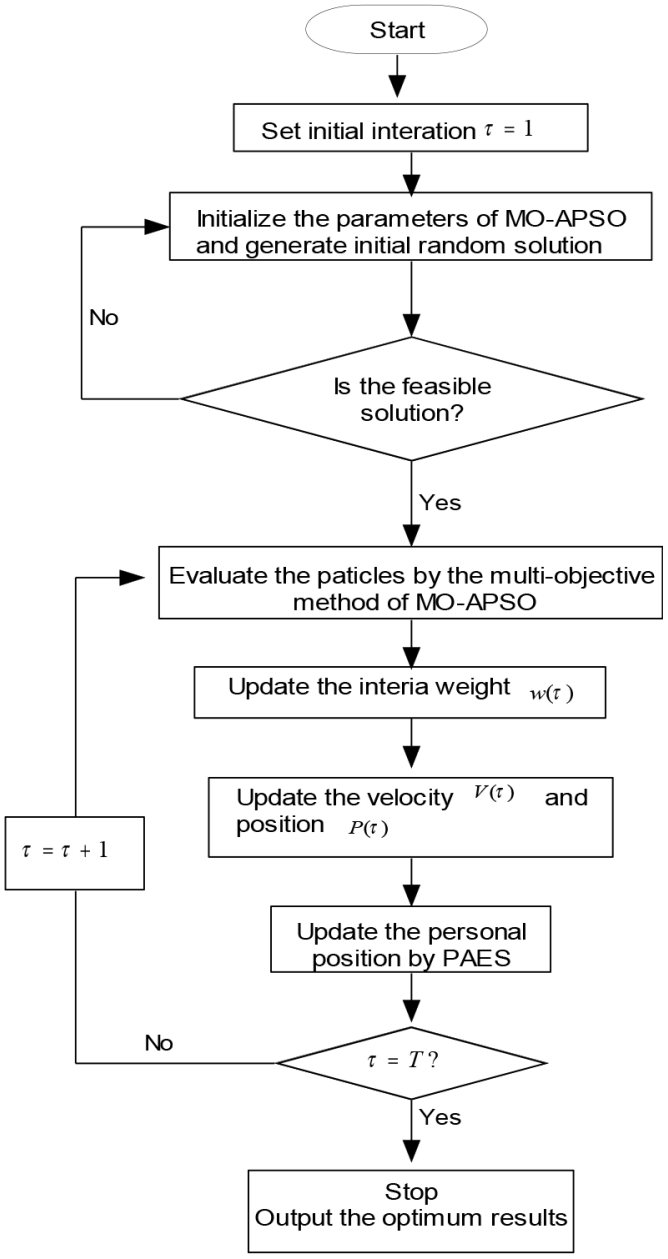

Figure 6. The procedure of MO-APSO 


\section{Application}

A practical supply chain application problem with quantity discount policy is considered in this section. Considering a small-scale but typical supply chain, which consists of two manufacturers $\left(m_{1}, m_{2}\right.$, i.e., $\left.m=2\right)$, two distribution centers $\left(d_{1}, d_{2}\right.$, i.e., $\left.d=2\right)$, two retailers $\left(r_{1}, r_{2}\right.$, i.e., $\left.r=2\right)$, and two products $\left(p_{1}, p_{2}\right.$, i.e., $\left.p=2\right)$, the time period is 3 (i.e., $t=3$ ). In this supply chain system, the first distribution center $\left(d_{1}\right)$, which is small scale but fast delivery service, can rapidly respond to the customer demand, but it also needs a high operational cost. Meanwhile, the second distribution center $\left(d_{2}\right)$, which is large scale but slow delivery service, but its operational cost is low because it can use the economies of scale to transport goods. The lead-time from each manufacturer to distribution center is 1 , and from distribution center to each retailer be 0 . The example of supply chain is depicted in Figure 7 .

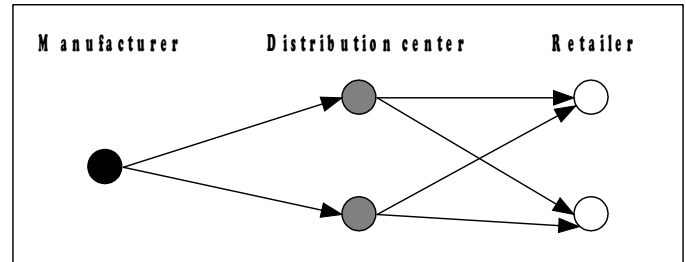

Figure 7. The example of typical supply chain

All the detailed data of supply chain system are gained from practical investigation. The bi-fuzzy variables are obtained based on previous data and experts' experience. In this case, product cost per unit $\tilde{\tilde{P}}_{\cos t_{m p}^{t}}$ and stock carrying cost $\tilde{\tilde{C}} \cos \mathbf{t}_{p d}^{t}$ are considered as multiple breakpoint function as shown in Figure 2 . The detailed information is shown in Table $1 \sim 5$.

\begin{tabular}{lll}
\hline & \multicolumn{1}{c}{ Product $1\left(p_{1}\right)$} & Product $2\left(p_{2}\right)$ \\
\hline \multirow{2}{*}{$t=1$} & $\tilde{\tilde{P}} \operatorname{cost} 11_{1}^{1}=\left(12.09, \tilde{\rho}^{1}, 14.81\right), \tilde{\rho}^{1}=(13.27,13.66,13.80)$ & $\tilde{\tilde{P}} \operatorname{cost12} 1=\left(4.21, \tilde{\rho}^{2}, 6.94\right), \tilde{\rho}^{2}=(5.51,5.63,5.88)$ \\
& $\tilde{\tilde{P}} \operatorname{cost} 11_{2}^{1}=\left(10.21, \tilde{\rho}^{3}, 11.82\right), \tilde{\rho}^{3}=(11.20,11.26,11.35)$ & $\tilde{\tilde{P}} \operatorname{cost} 12_{2}^{1}=\left(3.66, \tilde{\rho}^{4}, 4.02\right), \tilde{\rho}^{4}=(3.62,3.84,3.99)$ \\
& $\tilde{\tilde{P}}_{\operatorname{cost} 11_{3}^{1}=\left(7.60, \tilde{\rho}^{5}, 8.21\right), \tilde{\rho}^{5}=(7.95,8.06,8.22)}$ & $\tilde{\tilde{P}} \operatorname{cost} 12_{3}^{1}=\left(1.23, \tilde{\rho}^{6}, 2.38\right), \tilde{\rho}^{6}=(1.96,2.06,2.31)$ \\
\cline { 2 - 3 }$t=2$ & $\tilde{\tilde{P}} \operatorname{cost} 11_{1}^{2}=\left(9.45, \tilde{\rho}^{7}, 10.32\right), \tilde{\rho}^{7}=(9.64,9.89,9.99)$ & $\tilde{\tilde{P}} \operatorname{cost} 12_{1}^{2}=\left(6.85, \tilde{\rho}^{8}, 8.04\right), \tilde{\rho}^{8}=(7.41,7.54,7.86)$ \\
& $\tilde{\tilde{P}} \operatorname{cost} 11_{2}^{2}=\left(8.65, \tilde{\rho}^{9}, 9.46\right), \tilde{\rho}^{9}=(8.95,9.24,9.28)$ & $\tilde{\tilde{P}} \operatorname{cost} 12_{2}^{2}=\left(2.13, \tilde{\rho}^{10}, 2.65\right), \tilde{\rho}^{10}=(2.27,2.39,2.44)$ \\
& $\tilde{\tilde{P}} \operatorname{cost} 11_{3}^{2}=\left(7.01, \tilde{\rho}^{11}, 7.59\right), \tilde{\rho}^{11}=(7.03,7.10,7.16)$ & $\tilde{\tilde{P}} \operatorname{cost} 12_{3}^{2}=\left(3.51, \tilde{\rho}^{12}, 4.62\right), \tilde{\rho}^{12}=(3.59,3.66,3.74)$ \\
\cline { 2 - 4 }$t=3$ & $\tilde{\tilde{P}} \operatorname{cost} 11_{1}^{3}=\left(11.41, \tilde{\rho}^{13}, 12.34\right), \tilde{\rho}^{13}=(11.69,11.78,11.93)$ & $\tilde{\tilde{P}} \operatorname{cost} 12_{1}^{3}=\left(6.23, \tilde{\rho}^{14}, 7.19\right), \tilde{\rho}^{14}=(6.24,6.39,6.57)$ \\
& $\tilde{\tilde{P}} \operatorname{cost} 11_{2}^{3}=\left(8.10, \tilde{\rho}^{15}, 9.47\right), \tilde{\rho}^{15}=(8.61,8.79,8.96)$ & $\tilde{\tilde{P}} \operatorname{cost} 12_{2}^{3}=\left(2.06, \tilde{\rho}^{16}, 3.21\right), \tilde{\rho}^{16}=(2.49,2.78,3.06)$ \\
& $\tilde{\tilde{P}}_{\operatorname{cost} 11_{3}^{3}=\left(6.61, \tilde{\rho}^{17}, 6.97\right), \tilde{\rho}^{17}=(6.68,6.72,6.89)}$ & $\tilde{\tilde{P}} \operatorname{cost} 12_{3}^{3}=\left(4.68, \tilde{\rho}^{18}, 5.25\right), \tilde{\rho}^{18}=(4.86,4.93,5.01)$ \\
\hline
\end{tabular}

Table 1. Product cost per unit $\tilde{\tilde{P}} \cos t_{m p}^{t}$ (multiple breakpoint function as shown in Figure 2) 
Distribution center $1\left(d_{1}\right)$

Distribution center $2\left(d_{2}\right)$

$t=1,2,3 \quad p_{1} \quad \tilde{\tilde{T}} \cos t 1_{11}^{t}=\left(33.71, \tilde{\rho}^{19}, 38.32\right), \tilde{\rho}^{19}=(36.44,36.84,37.06) \quad \tilde{\tilde{T}}_{\cos t 1_{112}^{t}=\left(8.39, \tilde{\rho}^{20}, 9.68\right), \tilde{\rho}^{20}=(8.52,8.73,8.99)}$

$p_{2} \quad \tilde{\tilde{T}} \operatorname{cost} 1_{121}^{t}=\left(6.26, \tilde{\rho}^{21}, 7.91\right), \tilde{\rho}^{21}=(7.05,7.44,7.56) \quad \tilde{\tilde{T}} \operatorname{cost} 1_{122}^{t}=\left(19.34, \tilde{\rho}^{6}, 19.88\right), \tilde{\rho}^{22}=(19.42,19.51,19.74)$

Table 2. Transportation cost from manufacturer to distribution center $\tilde{\tilde{T}} \operatorname{cost} 1_{m p d}^{t}$

\begin{tabular}{|c|c|c|c|c|}
\hline \multirow{3}{*}{$t=1,2,3$} & & & Retailer $1\left(r_{1}\right)$ & Retailer $2\left(r_{2}\right)$ \\
\hline & $p_{1}$ & $d_{2}$ & $\begin{array}{l}\tilde{\tilde{T}} \operatorname{cost} 2_{111}^{t}=\left(1.04, \tilde{\rho}^{22}, 1.89\right), \tilde{\rho}^{22}=(1.43,1.51,1.67) \\
\tilde{\tilde{T}} \cos t 2_{121}^{t}=\left(9.32, \tilde{\rho}^{24}, 11.24\right), \tilde{\rho}^{24}=(10.54,10.69,11.01)\end{array}$ & $\begin{array}{l}\tilde{\tilde{T}} \operatorname{cost} 2_{112}^{t}=\left(4.62, \tilde{\rho}^{23}, 6.38\right), \tilde{\rho}^{23}=(5.94,6.18,6.72) \\
\tilde{\tilde{T}} \operatorname{cost} 2_{122}^{t}=\left(6.79, \tilde{\rho}^{25}, 8.87\right), \tilde{\rho}^{25}=(7.66,7.89,7.93)\end{array}$ \\
\hline & $p_{2}$ & $\begin{array}{l}a_{1} \\
d_{2}\end{array}$ & $\begin{array}{l}\tilde{\tilde{T}} \cos t 2_{211}^{t}=\left(1.85, \tilde{\rho}^{26}, 3.24\right), \tilde{\rho}^{26}=(2.05,2.16,2.44) \\
\tilde{\tilde{T}} \cos t 2_{221}^{t}=\left(3.22, \tilde{\rho}^{28}, 4.67, \tilde{\rho}^{28}=(3.95,4.08,4.16)\right.\end{array}$ & $\begin{array}{l}\tilde{\tilde{T}} \cos t 2_{212}^{t}=\left(12.65, \tilde{\rho}^{27}, 13.19\right), \tilde{\rho}^{27}=(12.88,12.93,13.00) \\
\tilde{\tilde{T}} \operatorname{cost} 2_{220}^{t}=\left(26.41, \tilde{\rho}^{29}, 28.11\right), \tilde{\rho}^{29}=(26.94,27.18,27.23)\end{array}$ \\
\hline
\end{tabular}

Table 3. Transportation cost from distribution center to retailer $\tilde{\tilde{T}} \operatorname{cost} 2_{p d r}^{t}$

\begin{tabular}{|c|c|c|c|}
\hline \multirow{9}{*}{$t=1,2,3$} & & Distribution center $1\left(d_{1}\right)$ & Distribution center $2\left(d_{2}\right)$ \\
\hline & $p_{1}$ & $\tilde{\tilde{C}} \operatorname{cost}_{11_{1}}^{t}=\left(1.46, \tilde{\rho}^{30}, 2.11\right), \tilde{\rho}^{30}=(1.47,1.55,1.59)$ & $\overline{\tilde{\tilde{C}} \operatorname{cost} t_{12}}{ }_{1}=\left(2.44, \tilde{\rho}^{31}, 4.36\right), \tilde{\rho}^{31}=(3.21,3.66,3.81)$ \\
\hline & & $\tilde{\tilde{C}} \operatorname{cost}_{11_{2}}^{t}=\left(0.95, \tilde{\rho}^{32}, 1.33\right), \tilde{\rho}^{32}=(0.96,0.98,1.03)$ & $\tilde{\tilde{C}} \operatorname{cost}_{12_{2}}^{t}=\left(2.38, \tilde{\rho}^{33}, 3.29\right), \tilde{\rho}^{33}=(2.41,2.46,2.63)$ \\
\hline & & $\tilde{\tilde{C}} \cos t_{11_{3}}^{t}=\left(0.83, \tilde{\rho}^{34}, 0.91\right), \tilde{\rho}^{34}=(0.86,0.89,0.90)$ & $\tilde{\tilde{C}} \operatorname{cost}_{12_{3}}^{t}=\left(2.08, \tilde{\rho}^{35}, 2.37\right), \tilde{\rho}^{35}=(2.11,2.24,2.29)$ \\
\hline & & $\tilde{\tilde{C}} \operatorname{cost}_{11_{4}}^{t}=\left(0.66, \tilde{\rho}^{36}, 0.78\right), \tilde{\rho}^{36}=(0.69,0.71,0.76)$ & $\tilde{\tilde{C}} \operatorname{cost}_{12_{4}}^{t}=\left(1.84, \tilde{\rho}^{37}, 1.98\right), \tilde{\rho}^{37}=(1.86,1.89,1.93)$ \\
\hline & $p_{2}$ & $\tilde{\tilde{C}} \operatorname{cost}_{21_{1}}^{t}=\left(18.33, \tilde{\rho}^{38}, 20.25\right), \tilde{\rho}^{38}=(19.31,19.45,19.81)$ & $\tilde{\tilde{\tilde{C}}} \operatorname{cost}_{22_{1}}^{t}=\left(4.36, \tilde{\rho}^{39}, 5.19\right), \tilde{\rho}^{39}=(4.81,4.89,5.02)$ \\
\hline & & $\tilde{\tilde{C}} \cos t_{21_{2}}^{t}=\left(16.62, \tilde{\rho}^{40}, 18.03\right), \tilde{\rho}^{40}=(16.78,16.96,17.11)$ & $\tilde{\tilde{C}} \operatorname{cost}_{22_{2}}^{t}=\left(4.07, \tilde{\rho}^{41}, 4.79\right), \tilde{\rho}^{41}=(4.16,4.19,4.28)$ \\
\hline & & $\tilde{\tilde{C}} \operatorname{cost}_{21_{3}}^{t}=\left(13.24, \tilde{\rho}^{42}, 15.66\right), \tilde{\rho}^{42}=(14.25,15.94,16.10)$ & $\overline{\tilde{C}} \operatorname{cost}_{22_{3}}^{t}=\left(3.16, \tilde{\rho}^{43}, 3.91\right), \tilde{\rho}^{43}=(3.19,3.54,3.62)$ \\
\hline & & $\tilde{\tilde{C}} \operatorname{cost}_{21_{4}}^{t}=\left(10.39, \tilde{\rho}^{44}, 12.98\right), \tilde{\rho}^{44}=(11.52,11.98,12.25)$ & $\tilde{\tilde{C}} \operatorname{cost}_{22_{4}}^{t}=\left(2.91, \tilde{\rho}^{45}, 3.15\right), \tilde{\rho}^{45}=(2.95,2.97,3.01)$ \\
\hline
\end{tabular}

Table 4. Stock carrying cost $\tilde{\tilde{C}} \operatorname{cost}_{p d}^{t}$ (multiple breakpoint function as shown in Figure 2)

\begin{tabular}{|c|c|c|c|}
\hline \multirow[t]{5}{*}{ The slope } & $\begin{array}{l}s_{P_{\operatorname{cost} 11^{1}}}=-0.06 \\
s_{P_{\text {cost } 121} 1}=-0.30\end{array}$ & $\begin{array}{l}s_{2}^{P_{\text {cost } 11^{1}}}=-0.75 \\
s_{P_{\text {cost } 121}}=-0.42\end{array}$ & \\
\hline & ${ }^{s_{1}} \cos _{11}^{t}=-0.27$ & $s_{2}{ }_{C \cos t}^{t}=-0.76$ & $s_{3} \operatorname{Ccost}_{11}^{t}=-0.88$ \\
\hline & ${ }^{s_{1}} \operatorname{Ccost}_{12}^{t}=-0.32$ & $s_{2}{ }_{C \cos t}^{t}{ }_{12}=-0.09$ & ${ }^{s_{3}}{ }_{C \operatorname{cost}} t_{12}^{t}=-0.25$ \\
\hline & $s_{1}{ }_{C \cos t}^{t}=-0.32$ & $s_{2}{ }_{C \cos t}^{t}=-0.09$ & $s_{3}{ }_{C \cos t}^{t}{ }_{21}=-0.25$ \\
\hline & ${ }^{s_{1}}{ }_{C \operatorname{cost}}^{t}{ }_{22}=-0.32$ & ${ }^{s_{2}}$ c cost $_{22}^{t}=-0.09$ & ${ }^{s_{3} C_{\operatorname{cost}}{ }_{22}^{t}}=-0.25$ \\
\hline maximum inventory capacity & $M I C_{m}=48$ & $M I C_{d}=26$ & $M I C_{r}=32$ \\
\hline Maximum supplied level & $S_{m p}^{t}(\max )=46$ & & \\
\hline Maximum inventory level for $p$ and $d$ & $I_{p d}^{t}(\max )=55$ & & \\
\hline Possibility level and expected value index & $\alpha_{1}=0.8$ & $\alpha_{2}=0.5$ & $\lambda=0.7$ \\
\hline
\end{tabular}

Table 5. Others parameters 


\subsection{The results}

The parameters of MO-APSO for the multiple objective SCM problem are: swarm size popsize $L=50$, iteration $\max T=100$, personal and global best position acceleration constant $c_{p}=c_{g}=2$, inertia weight $w(1)=0.4, w(T)=0.9$. After dealing with the bi-fuzzy variables by the hybrid crisp approach in section 3, we use Matlab 7.0 and Visual $C++$ language on an Inter Core I7 M370, $2.40 \mathrm{GHz}$, with $2048 \mathrm{MB}$ memory, and take the data into the computer program, the optimal solution of SCM model is generated by MO-APSO (see Figure 8).

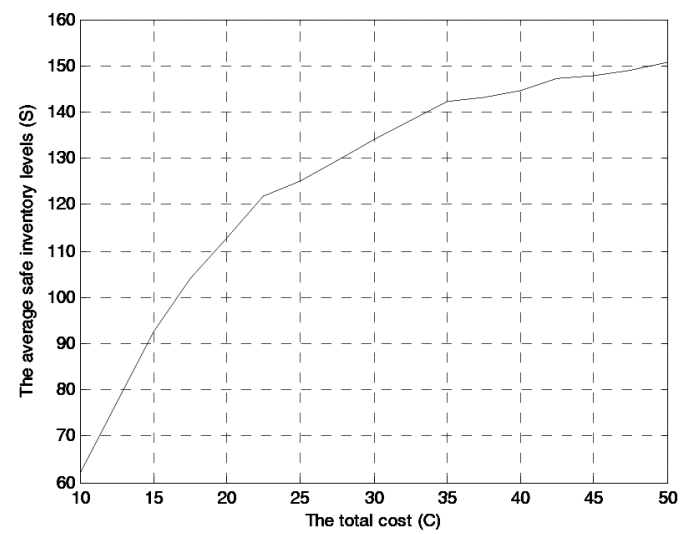

Figure 8. The Pareto optimal solutions

Following Figure 8, DMs could choose the satisfactory scenarios from these pareto-optimal solutions according to the actual situation. For example, if DMs determine that the objective of total cost is the more important factor, they may allow a decreased average safe inventory levels. Thus, they would choose the far left pareto-optimal solutions.

\subsection{Sensitivity analysis}

In order to evaluate the effect of variations in model parameters, sensitivity analysis is performed. For illuminating the sensitivity clearly, we changed the value of $\alpha_{2}, \alpha_{1}$, and $\lambda$ in turn, then compared the corresponding results to analyze the effect of each parameter. This work can provide the necessary information to the DMs for choosing the value of each parameter when considering the actual situation.

First, let $\alpha_{1}$ still be 0.8 as before, and $\alpha_{2}=0.4,0.3,0.2$, respectively. Then, we get Figure 9 . Subsequently, let $\alpha_{2}$ still be 0.5 , and $\alpha_{1}=0.7,0.6,0.5$, respectively. Then we obtain Figure 10 . 
Finally, let $\alpha_{1}$ still be 0.8 and $\alpha_{2}$ still be $0.5, \lambda=0.6,0.5,0.4$, respectively. Then we gain 11 . Based on the sensitivity analysis of the results, the bi-fuzzy nonlinear MODM model for SCM is proved to be sensitive to the possibility level $\alpha_{1}$.

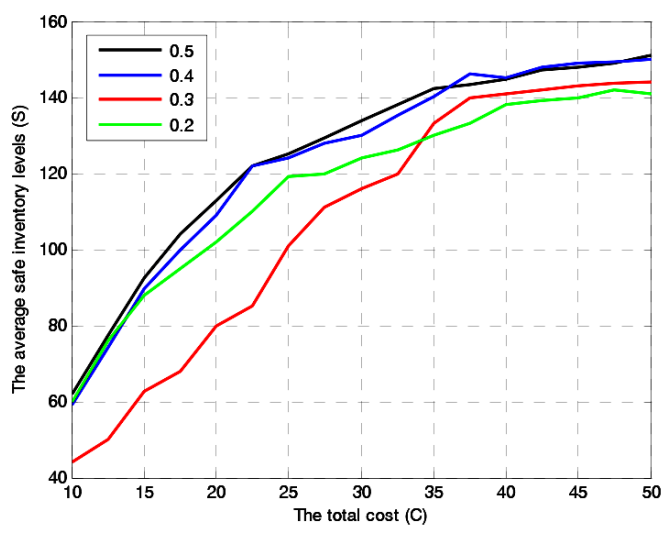

Figure 9. The sensitivity of $\alpha_{2}$

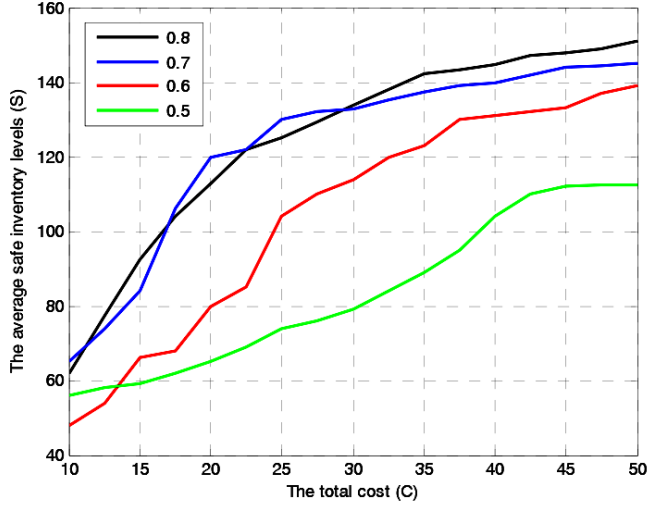

Figure 10. The sensitivity of $\alpha_{1}$

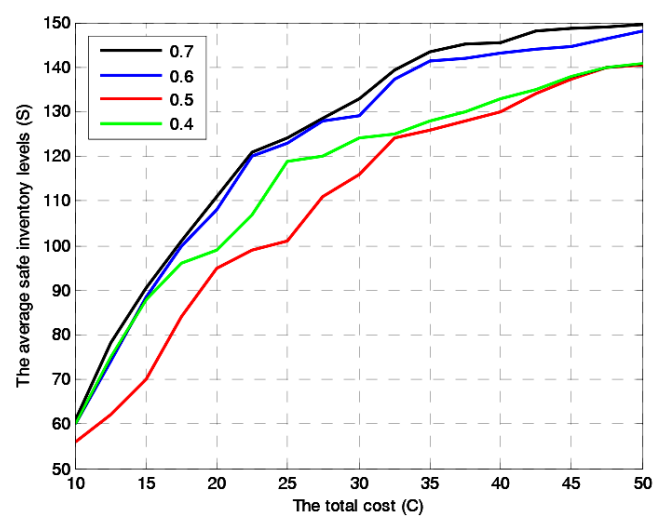

Figure 11 . The sensitivity of $\lambda$

In practice, the DMs can change the parameters $\alpha_{1}, \alpha_{2}$ and $\lambda$ to obtain the different solutions under the different levels of parameters. The solutions reflect different optimistic-pessimistic attitudes for uncertainty and different predictions of possibility levels.

\section{Conclusions and further research}

Considering the complex fuzzy environment, a nonlinear MODM model for SCM with quantity discount policy is presented in this paper. The bi-fuzzy variables are transformed into the trapezoidal fuzzy variables by the DMs's degree of optimism $\alpha_{1}$ and $\alpha_{2}$, which are de-fuzzified by the expected value index $\lambda$ subsequently. In order to solve the equivalent crisp model, MO-APSO algorithm is designed to obtain the optimal solution. For illustrating the 
effectiveness, the proposed model and algorithm are applied to a typical example of SCM problem.

The main contributions of this study are as follows: (1) The bi-fuzzy variable is employed in the nonlinear MODM model of SCM to characterize the hybrid uncertain environment, and this work is original. (2) The proposed model is transferred to an equivalent crisp on by the DMs's degree of optimism and the expected value index. For solving the complex model, MO-APSO is designed as the solution method. (3) The study focuses on the SCM under complex fuzzy environment in SCM, which has a great practical significance.

The area for future research has two aspects: firstly, more realistic factors and constraints for SCM with complex hierarchical organization structure should be considered; secondly, more efficient heuristic methods should be designed to solve this nonlinear MODM model. Both areas are important and worth the concern.

\section{Acknowledgment}

This research was sponsored by Jiangsu Provincial Natural Science Foundation of China (Grant No. BK20130753), Fundamental Research Funds for the Central Universities (Grant No. JGQN1403, No. 30920130132012), "985" Program of Sichuan University "Innovative Research Base for Economic Development and Management". We would like to give our great appreciates to all editors who contributed this research.

\section{References}

Al-e-hashem, S., Malekly, H., \& Aryanezhad, M. (2011). A multi-objective robust optimization model for multi-product multi-site aggregate production planning in a supply chain under uncertainty. International Journal of Production Economics, 134, 28-42.

http://dx.doi.org/10.1016/j.ijpe.2011.01.027

Altiparmak, F., Gen, M., Lin, L., \& Paksoy, T. (2006). A genetic algorithm approach for multiobjective optimization of supply chain networks. Computers \& Industrial Engineering, 51(1), 196-215. http://dx.doi.org/10.1016/j.cie.2006.07.011

Arikan, F. (2013). A fuzzy solution approach for multi objective supplier selection. Expert Systems with Applications, 40(3), 947-952. http://dx.doi.org/10.1016/j.eswa.2012.05.051

Chen, C., \& Lee, W. (2004). Multi-objective optimization of multi-echelon supply chain networks with uncertain product demands and prices. Computers \& Chemical Engineering, 28(6-7), 1131-1144. http://dx.doi.org/10.1016/j.compchemeng.2003.09.014 
Coello, C., \& Lechuga, M. (2002). MOPSO: A proposal for multiple objective particle swarm optimization. In Proceedings of the 2002 Congress on Evolutionary Computation, 1051-1056.

Dubois, D., \& Prade, H. (1988). Possibility Theory: An Approach to Computerized Processing of Uncertainty. New York: Plenum. http://dx.doi.org/10.1007/978-1-4684-5287-7

Garg, H., \& Sharma, S. (2013). Multi-objective reliability-redundancy allocation problem using particle swarm optimization. Computers \& Industrial Engineering, 64(1), 247-255. http://dx.doi.org/10.1016/j.cie.2012.09.015

Giannoccaro, I., Pontrandolfo, P., \& Scozzi, B. (2003). A fuzzy echelon approach for inventory management in supply chains. European Journal of Operational Research, 149(1), 185-196. http://dx.doi.org/10.1016/S0377-2217(02)00441-1

Kennedy, J., \& Eberhart, R. (1995). Particle swarm optimization. In Proceedings of the IEEE Conference on Neural Networks, 1942-1948. Piscataway: IEEE Service Center.

Kennedy, J., \& Eberhart, R. (2001). Swarm Intelligence. Morgan Kaufmann.

Knowles, J., \& Corne, D. (2000). Approximating the nondominated front using the pareto archived evolution strategy. Evolution Computation, 8, 149-172.

http://dx.doi.org/10.1023/A:1013771608623

Liu, B. (2002). Toward fuzzy optimization without mathematical ambiguity. Fuzzy Optimization and Decision Making, 1(1), 43-63. http://dx.doi.org/10.1023/A:1013771608623

Liu, Y., \& Xu, J. (2006). A class of bifuzzy model and its application to single-period inventory problem. World Journal of Modelling and Simulation, 2(2), 109-118.

Luhandjula, M. (1987). Multiple objective programming problems with possibility coefficients. Fuzzy Sets and Systems, 21, 135-145. http://dx.doi.org/10.1016/0165-0114(87)90159-X

Nahmias, S. (1978). Fuzzy variables, Fuzzy Sets and Systems, 1, 97-110.

http://dx.doi.org/10.1016/0165-0114(78)90011-8

Shi, Y., \& Eberhart, R. (1998). Particle swarm optimization. In Proc. IEEE Int. Conf. on Neural Networks, 69-73.

Simchi-Levi, D., Kaminsky, P., \& Simchi-Levi, E. (2000). Designing and Managing the Supply Chain. New York: Irwin McGraw-Hill.

Tabrizi, B., \& Razmi, J. (2013). Introducing a mixed-integer non-linear fuzzy model for risk management in designing supply chain networks, Journal of Manufacturing Systems, 32(2), 295-307. http://dx.doi.org/10.1016/j.jmsy.2012.12.001 
Tavakkoli-Moghaddam, R., Azarkish, M., \& Sadeghnejad-Barkousaraie A. (2011). A new hybrid multi-objective Pareto archive PSO algorithm for a bi-objective job shop scheduling problem. Expert Systems with Applications, 38(9), 10812-10821. http://dx.doi.org/10.1016/j.eswa.2011.02.050

Torabi, S., \& Hassini, E. (2008). An interactive possibilistic programming approach for multiple objective supply chain master planning. Fuzzy Sets and Systems, 159(2), 193-214. http://dx.doi.org/10.1016/j.fss.2007.08.010

Wang, J., \& Shu, Y. (2008). Fuzzy decision modeling for supply chain management. Fuzzy Sets and Systems, 150(1), 107-127. http://dx.doi.org/10.1016/j.fss.2004.07.005

Wei, C., Liang, G., \& Wang, M. (2007). A comprehensive supply chain management project selection framework under fuzzy environment. International Journal of Project Management, 25(6), 627-636. http://dx.doi.org/10.1016/j.ijproman.2007.01.010

Xu, J., \& Liu, Y. (2008). Multi-objective decision making model under fuzzy random environment and its application to inventory problems. Information Sciences, 178, 2899-2914. http://dx.doi.org/10.1016/j.ins.2008.03.003

Xu, J., \& Yan, F. (2011). A multi-objective decision making model for the vendor selection problem in a bifuzzy environment. Expert Systems with Applications, 38(8), 9684-9695. http://dx.doi.org/10.1016/j.eswa.2011.01.168

Xu, J., \& Zhou, X. (2011). Fuzzy-Like Multiple Objective Decision Making. Berlin Heidelberg: Springer-Verlag.

Yan, L. (2009). Risk Curve and Bifuzzy Portfolio Selection. Journal of Mathematics Research, 1(2), 193-198. http://dx.doi.org/10.5539/jmr.v1n2p193

Zadeh. L. (1965). Fuzzy sets. Information and Control, 8, 338-353.

http://dx.doi.org/10.1016/S0019-9958(65)90241-X

Zadeh. L. (1978). Fuzzy sets as a basis for a theory of possibility. Fuzzy Sets and Systems, 1, 3-28. http://dx.doi.org/10.1016/0165-0114(78)90029-5

Zhang, G., Shao, X., Li, P., \& Gao, L. (2009). An effective hybrid particle swarm optimization algorithm for multi-objective flexible job-shop scheduling problem. Computers \& Industrial Engineering, 56(4), 1309-1318. http://dx.doi.org/10.1016/j.cie.2008.07.021

Journal of Industrial Engineering and Management, 2014 (www.jiem.org)

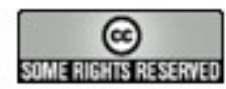

Article's contents are provided on a Attribution-Non Commercial 3.0 Creative commons license. Readers are allowed to copy, distribute and communicate article's contents, provided the author's and Journal of Industrial Engineering and Management's names are included. It must not be used for commercial purposes. To see the complete license contents, please visit http://creativecommons.org/licenses/by-nc/3.0/. 